\title{
Editorial: Achieving Water-Energy-Food Nexus Sustainability: A Science and Data Need or a Need for Integrated Public Policy?
}

\author{
Rabi Mohtar ${ }^{1,2 *}$, Richard George Lawford ${ }^{3}$ and Jill A. Engel-Cox ${ }^{4}$ \\ ${ }^{1}$ TEES Research, Texas A\&M University, College Station, TX, United States, ${ }^{2}$ Faculty of Agricultural and Food Sciences, \\ American University of Beirut, Beirut, Lebanon, ${ }^{3}$ School of Computer, Mathematical and Natural Sciences, Morgan State \\ University, Baltimore, MD, United States, ${ }^{4}$ National Renewable Energy Laboratory, Joint Institute for Strategic Energy \\ Analysis, Golden, CO, United States
}

Keywords: water, global change, environmental policy, future sustainability, water-energy-food nexus

\author{
Editorial on the Research Topic
}

Achieving Water-Energy-Food Nexus Sustainability: A Science and Data Need or a Need for Integrated Public Policy?

OPEN ACCESS

Edited by:

Rebecca Elizabeth Tharme, Riverfutures Ltd, United Kingdom

Reviewed by:

Eline Boelee,

Deltares, Netherlands

${ }^{*}$ Correspondence: Rabi Mohtar mohtar@tamu.edu; mohtar@aub.edu.lb

Specialty section: This article was submitted to

Freshwater Science,

a section of the journal

Frontiers in Environmental Science

Received: 15 November 2019 Accepted: 16 July 2020 Published: 28 August 2020

Citation:

Mohtar R, Lawford RG and Engel-Cox JA (2020) Editorial: Achieving Water-Energy-Food Nexus Sustainability: A Science and Data Need or a Need for Integrated Public

Policy? Front. Environ. Sci. 8:132. doi: 10.3389/fenvs.2020.00132

\section{INTRODUCTION}

The benefits of addressing the water, energy, and food sectors in an integrated manner are gaining significant recognition. An integrated approach can provide improved resource use efficiencies, more coherent environmental policies, and an overall strategy for achieving sustainability in the three sectors, as outlined in the United Nations Sustainable Development Goals (SDG) 2 (Food), 6 (Water), and 7 (Energy). Societies are concerned with ensuring food security, avoiding wars over water, and creating opportunities by ensuring access to energy. To be effective, however, this approach needs to be adopted by all segments of society including government, the private sector, and civil society and must be reinforced by appropriate management and planning approaches.

This special issue identifies different approaches that are either being conceptualized or tested to support the Water-Energy-Food (WEF) Nexus approach. The articles contribute to answering the question, "Is achieving Water-Energy-Food (WEF) Nexus Sustainability a science and data need or an integrated public policy need?" In either case, both natural and social scientists need to combine their efforts to address interdisciplinary science issues and integrated policy approaches. The papers in this special issue explore the ways in which science, data, and policy development could help define integrative principles and policies for the three sectors. This approach could expand beyond the water, energy, and food sectors to include health, environment, trade, commerce, and international assistance, thereby providing broad support to the SDGs. Moreover, this issue demonstrates that data combined with new technologies (tools and models) can support better decision-making when adopted by governments and the management cadre for each of these sectors.

The issue includes 25 papers each designated as a specific type of paper by their authors. The papers include 3 policy and practice review [PPR] papers, 2 hypothesis and theory [HT] papers, 2 review $[\mathrm{R}]$ papers, 1 systematic review [SR] paper, 1 perspective $[\mathrm{P}]$ paper, and 16 original research $[\mathrm{OR}]$ papers. This editorial provides a summary of main messages in the special issue with a synthesis of the 25 papers and offers a perspective on gaps in the WEF Nexus science and 
policy domains. Although no one paper answered all aspects of the question posed in the title, when considered together the papers provided an overall perspective. That perspective is summarized in the last paragraph of this editorial.

\section{SYNTHESIS OF SPECIAL ISSUES AND LESSONS LEARNED}

The papers all had a primary focus, which allowed them to be grouped into the following four categories in order to better review their findings:

i. Applications and gaps in governance

ii. Availability of new science, observations, and information technologies

iii. Pragmatic solutions to regional WEF Nexus problems

iv. Approaches to effectively moving scientific information and new technologies into governance and public action

The key findings from the papers in each category are summarized below. It should be noted that in addition to their primary focus, some papers also addressed issues in other categories. To keep the review focused, however, we have based our conclusions on what we believe is the primary contribution of each paper.

\section{Applications and Gaps in Governance}

Although some approaches are directed from the top, others consider the views and concerns of communities and sectors involved. Wiegleb and Bruns argue for cross-sectoral Nexus approaches for resource governance and Nexus discourse. They conclude that the traditional utilitarian approach to resource governance should be supplemented by a multi-perspective approach that addresses the societal and environmental factors affecting WEF Nexus dynamics.

There are different views of how governance should be implemented to manage integrated resources like water, energy, and food. Often the needs of the WEF Nexus approach are most evident when conflicts exist in management and governance structures. Based on a review of scientific literature related to the WEF Nexus as a framework for resource security, Simpson and Jewitt review interpretations of the WEF Nexus concept and discuss challenges associated with integrating and optimizing its multi-centric components. Liu et al. investigate the competition for the allocation of critical resources among the water, energy, and food sectors in China and conclude that WEF Nexus approaches are needed to lessen conflict among ministries in ways that support sustainable water management and food production while conserving the potential for hydropower generation.

Water's importance in the WEF Nexus and in economic growth more generally is an important policy issue. Markantonis et al. address this in the context of the Mediterranean region, where ongoing water scarcity is further exacerbated by climate change. The authors explore how implementing the WEF Nexus can support economic growth under the anticipated water constraints. Land is another critical resource that constrains WEF
Nexus sustainability in many countries. Based on land use issues in South Africa, Simpson et al. highlight the competition over land use (food/water securities vs. mineral rights and extractions) and emphasize the critical need for WEF Nexus science and data to influence integrated public policy and land use decisions.

A number of regional studies demonstrate that similar challenges and issues are resolved very differently in various regions because of governance. Although geography is important, a specific region may face greater problems for different reasons. Hoff et al. found that the absence of a clear definition of the WEF Nexus in the Middle East and North Africa limits the ability to communicate the concept and often hinders the adoption of WEF Nexus principles. Scenario analysis is an important aspect of both policy and technology that support better communication.

Dombrowsky and Hensengerth address the roles of investors, governance, and planning structures (joint vs. individual) for riparian basins. The roles of regional organizations in joint planning and consensus development should be included in the pathways for solutions.

Education will be an important tool for developing and promoting an understanding of the WEF Nexus (or FEW Nexus, in National Science Foundation parlance) and its implications. Rodríguez et al. review options for educational programs, proposing a curriculum outline that includes typical WEF Nexus challenges and toolbox modules for addressing them. The authors emphasize the need for transdisciplinary education and training.

\section{Availability of New Science, Observations, and Information Technologies}

Evidence-based decision-making in support of the WEF Nexus relies on scientific understanding and related information, which in turn is based on data and observations, and models and tools for analysis. New capabilities to observe at high resolution over global scales are changing the ability of the science community to support integrated WEF Nexus planning. Giupponi et al. explicitly identify the need to define assessment procedures that go beyond national or regional aggregation. They argue for "zooming in" to local phenomena in order to identify the policies and technologies that will provide solutions at the scales where production occurs and meaningful decisions can be made.

Through a diverse set of case studies using NASA Earth observations, McNally et al. demonstrate the benefits of these observational systems for the Water-Food Nexus. They also demonstrate the value of applying these data to issues in the water-food component of the WEF Nexus and the benefits of stakeholder interactions to data providers to help transform Earth observations into more accurate, timely, and relevant products and information. Bruss et al. demonstrate how Gravity Recovery and Climate Experiment (GRACE) data can be analyzed to provide key predictors that can help inform policies for water management in areas such as water supply and water use. Understanding the context of these policies is important for determining the optimum role of data. 
Lawford hypothesizes that readily available data and information can be used to promote integrated resource planning. He proposes a series of steps for designing, implementing, and testing an integrated data and information system in a WEF Nexus decision-making environment. He also emphasizes the critical roles of observations data, information, and modern technology in facilitating the paradigm shifts needed to advance WEF Nexus approaches.

Kurian et al. address the underlying siloes, trade-offs, and synergies of the Nexus and advocate for specialized longterm multi-sector observatories located where specific issues are expected. Local measurements, qualitative observations, and data obtained regularly over a long period can be used in combination with policy instruments (guidelines, notifications, standards, circulars, and directives) to provide information for better decision-making on a local or regional basis and to support the valorization of data and assess methodological assumptions.

\section{Pragmatic Solutions to Regional Wef Nexus Problems}

A number of potential practical solutions were elaborated, many of them tailored for specific geographical areas or a specific aspect of the WEF Nexus. Urbanization is often accompanied by the rapid expansion of urban populations in areas without proper infrastructure, urban ecosystems, and burgeoning populations that threaten food security and raise the risk of food crisis events, particularly in the developing world as Davies and Garrett demonstrate. They discuss the role of smart technology and identify 12 innovative technology platforms to promote urban food ecosystems. When linked to value chains, these platforms can lead to both increased entrepreneurial opportunities and greater efficiency of resource use. Schulterbrandt Gragg et al. describe urbanizing socioecological systems from a policy perspective and offer an iterative, multidimensional model that provides new opportunities for solutions and stakeholder involvement in assessing options.

Shumilova et al. discuss water transfer megaprojects and the challenges of using existing hydrological models to assess the impacts of these projects and to recommend design criteria. These hydrological models currently do not explicitly include ecological, social, and economic factors and are therefore of limited value for impact assessment. More comprehensive models should be used to ensure maximum relevance for decision makers and operational managers. A similar conclusion is made by Givens et al. who report on decision-making in the Columbia River basin. Their study, which is based on the physical models typically used in river basin management, identifies limitations with the current approach. The authors call for the inclusion of social aspects (inequality, power, social justice) in the set of models used to address river basin planning.

In additional to traditional approaches, new technologies and methodologies are critical to WEF Nexus security. Haskett et al. discuss the potential use of the decaying leaves of Faidherbia albida trees in Ethiopia to fertilize nearby crops. In tropical countries with suitable climates this method could reduce the production and transportation of chemical fertilizers and reduce regional greenhouse gas emissions. Alemneh et al. demonstrate the importance of monitoring cropland conversions in order to quantitatively assess their impacts on the local production of food and water, energy, and food consumption.

\section{Approaches to Effectively Moving Scientific Information and New Technologies Into Governance and Public Action}

Trade-offs are an essential aspect of managing WEF resources. The key to future sustainable development in these resource sectors involves balancing supply and demand at all scales and under all environmental conditions. Linking WEF Nexus activities to the relevant SDGs would facilitate the development of joint monitoring and modeling strategies. Efforts in one sector can synergistically benefit the two other sectors. Fader et al. developed and applied a quantitative methodology to provide a replicable way of identifying potential synergies and trade-offs in the implementation of the food, water, and energy SDG targets. By extension, the WEF Nexus could be implemented using a similar assessment tool.

Strategies are needed to address tradeoffs, especially when it is difficult to bring entrenched interests together to discuss compromise. Frameworks such as that discussed by Allam and Eltahir in their study of the Nile River basin use optimization models to produce a range of options for the allocation of land and water resources. These model outputs are analyzed to produce problem-solving scenarios with stakeholders when cooperation is difficult to obtain.

Stakeholder perspectives and interactions are highlighted by Bielicki et al. who conclude that, with regard to WEF Nexus sustainability, the importance and use of science, data, and integrated policy depends on the context in which the stakeholders operate in the WEF Nexus domain. Linking strategies that rely on stakeholder interactions are outlined by Yung et al., who found that the strategic use of the characterization and navigation of Nexus uncertainties could facilitate integrated risk assessment. The authors stress the importance of stakeholder engagement for obtaining local knowledge of cultural and economic considerations that could affect the implementation of broad-scale WEF Nexus policies.

Holistic WEF Nexus frameworks are reviewed by Kulat et al. They show how these models offer insights into sustainable (and unsustainable) scenarios that are critical to developing policies that will preserve WEF resources without disrupting economic well-being and the health of ecosystems. Taniguchi et al. also present a holistic model by assessing the effectiveness of a Japanese government program that subsidizes farmers for ponding water on their fields. Subsidized water ponding leads to groundwater recharge, reduced energy use, and increased food production. This tool is one of a number available for cities, states, and countries to evaluate their policy interventions on WEF Nexus sustainability.

As this special issue demonstrates, there are many tools available to help engage stakeholders. The challenge is to inform stakeholders, and to provide timely and relevant information to 
enable them to make better decisions for themselves and for WEF Nexus sustainability. Increasingly reliable modeling produces improved scenarios for policy and technology. Discussing with stakeholders outcomes that support long-term sustainability could be a good way to encourage much broader awareness of the WEF Nexus and the importance of its sustainability.

\section{GENERAL FINDINGS AND RESEARCH GAPS}

This special issue shows that both the socioeconomic and physical dimensions of the WEF Nexus need to be addressed jointly-but the integration of these approaches requires a considerable amount of work and research. There are many challenges to bridging the social and the physical sciences as they relate to the WEF Nexus. To make progress on the WEF Nexus goals and to support SDG implementation, both communities need to develop mutually beneficial research strategies. Collaborations could be enhanced by exploring opportunities for joint WEF Nexus and SDG monitoring systems and other options.

Other approaches that could advance WEF Nexus implementation include tools and models for the WEF Nexus, relevant interdisciplinary research approaches, indices, and monitoring systems that can support governance and evidencebased decision-making. Addressing siloed resources and expertise-and the current lack of policy coherence-demands "out-of-the-box" thinking and more integrated approaches to planning and management.

Integrated modeling tools are essential to produce relevant outputs, including analyses and scenarios that support the development of policies on sustainable consumption. Such policies are informed by the strong links between issues like fossil fuel extraction and water quality or energy subsidies, which can lead to overexploitation of groundwater for food production. Models play a key role in the generation of scenarios, but their scientific underpinnings must be sound for the resulting scenarios to be credible. While integrated models can help clarify and quantify the trade-offs of various scenarios, the limitations and appropriate applications and uncertainties of models must be understood.

Currently, modeling tools are not optimally used by policy makers because there is a lack of interactions among scientists, engineers, and practitioners in the water, energy, and agricultural sectors to develop an appreciation for the value of integration. Siloed science and governance approaches contribute to the lack of informed and optimized multi-sector decisions. This challenge can be overcome, at least in part, with interdisciplinary work and convergent scientific and applications infrastructure. In particular, collaborative efforts among experts from each sector to increasingly integrate sector-specific models into comprehensive WEF Nexus models could be an important step in overcoming these siloed effects.
As a result of their complexity, WEF Nexus issues present interconnected challenges where the best solutions to problems in one sector often are achieved by working collaboratively with the other sectors. For example, some water issues can only be solved through collaboration with the food and energy sectors, and vice versa. Only interdisciplinary collaboration can result in truly encompassing discussions that identify common goals for the various consumers, experts, and policy makers. These collaborations should be based on an understanding of the science and short- and longterm policy implications of each option involved in WEF Nexus sustainability.

Better systems for acquiring and processing data, and rapidly disseminating data products and information for planning and decision-making, are essential. Many of the components for such a system are available "off the shelf," but each system needs to be co-designed or at least coordinated with careful attention to the needs, perceptions, and assessments of product utility by stakeholders and users.

This special issue demonstrates that there are a large number of innovative applications of special techniques, local practices, and sophisticated tools and models that are all available for addressing the WEF Nexus issues. There is no single framework to structure and disseminate this information. It would be beneficial to have an inventory for the many users that would benefit from this information. Some of the papers in this collection emphasize two-sector interactions such as water and food or energy and food. These papers generally addressed the WEF Nexus as a conceptual framework rather than an analytical approach to sustainability. Joint research that engages the expertise and information available for all three sectors is needed.

As part of efforts to develop stronger commitments for the implementation of WEF Nexus principles, national meetings feeding into an international strategy for the WEF Nexus could advance collaboration and integrated approaches to development and applications. One or more high-profile events with policy engagement could advance the development of a WEF Nexus planning approach by many nations. Such an event could focus on the development and endorsement of a roadmap or implementation plan for the WEF Nexus as one of its principal outputs. A model for this event could be the 1993 Dublin International Conference on Water and the Environment.

Human security and ecosystem services need to be included in WEF Nexus decision-making. New measures and data streams could be developed for agricultural and ecosystem services outputs so that statistics such as tons of produce per hectare would be supplemented with other measures such as concentrations of nutrients, proteins, water and energy footprints, energy production/use, and other environmental parameters. People may be more supportive of WEF Nexus principles if they see how important aspects of each sector are changing. 
The papers raised a number of science questions that also relate to developing new paradigms. Some of these questions include the following:

- Given the capabilities of satellites to provide the spatial distribution of water quality variables such as phytoplankton, why are measures of ambient water quality not having a greater role in decision-making? For example, non-point source pollution from nitrogen and phosphorus arising from fertilizers and enhanced by irrigation is a critical problem that needs to be monitored regularly in the WEF Nexus. How can end-point measurements be supplemented by ambient measures to develop indicators that can assess WEF interlinkages?

- How can hydrological models incorporate more energy, food, environmental, and socioeconomic factors to produce scenarios that inform the public and motivate action?

- In many areas, one or more components of the WEF Nexus will be very sensitive to climate change. Several papers referenced hydropower, a major source of clean energy, that nonetheless is vulnerable in areas where prolonged dry periods can persist. In a similar way agricultural irrigation is under high risk and uncertainty due to climate change impacts on water availability in certain regions. How will climate change impact the WEF Nexus and water-stressed regions?

- In the context of meeting water needs, including those outlined in SDG 6, how can we ensure that recycled water fully alleviates water supply deficits without affecting irrigation water quality and food security and safety? In turn, how can we make better use of energy recovery systems in wastewater facilities?

Although the title of this paper suggests WEF Nexus sustainability could be achieved solely by either a policy approach or the use of science and technology, papers in this special issue have shown that both public policy and science/data are necessary for WEF Nexus sustainability. This assessment concludes that a blend of monitoring data, coordinated research, public policy, and governance are needed at the national and global scales to help set goals to encourage the sectors to work together to address broader integration as needed. Scientific understanding and data are needed to support evidence-based decision making to address these problems within the context of the rules set out by governance decisions. Policy must be adopted that not only reflects regional priorities, but also supports global public policy such as sustainable development. When coordinated, data and science can provide support for decisions at all scales from local transient problems to macroscale research and the observational systems needed to monitor the overall status of the WEF Nexus on a global basis.

\section{AUTHOR CONTRIBUTIONS}

All authors listed have made a substantial, direct and intellectual contribution to the work, and approved it for publication.

\section{FUNDING}

$\mathrm{RL}$ acknowledged the support he received while working on this publication from USRA Cooperative Agreement 05700-01-02.

Conflict of Interest: The authors declare that the research was conducted in the absence of any commercial or financial relationships that could be construed as a potential conflict of interest.

Copyright (c) 2020 Mohtar, Lawford and Engel-Cox. This is an open-access article distributed under the terms of the Creative Commons Attribution License (CC BY). The use, distribution or reproduction in other forums is permitted, provided the original author(s) and the copyright owner(s) are credited and that the original publication in this journal is cited, in accordance with accepted academic practice. No use, distribution or reproduction is permitted which does not comply with these terms. 